\title{
Clinical evaluation of embolization of the superior vesical prostatic artery for treatment of benign prostatic hyperplasia: a single-center retrospective study
}

\author{
Zhilei Qiu ${ }^{1}$, Changcun Zhang ${ }^{1}$, Xinsheng Wang ${ }^{2}$, Kai Cheng ${ }^{1}$, Xin Liang ${ }^{1}$, Dawen Wang ${ }^{1}$, Sichuan Hou ${ }^{1}$, Xinsheng Wang ${ }^{3}$ \\ ${ }^{1}$ Department of Urology, The Affiliated Qingdao Municipal Hospital of Qingdao University, Qingdao, China \\ ${ }^{2}$ Department of Urology, Tianjin First Center Hospital, Tianjin, China \\ ${ }^{3}$ Department of Urology, The Affiliated Hospital of Qingdao University, Qingdao, China
}

Videosurgery Miniinv 2017; 12 (4): 409-416

DOI: https://doi.org/10.5114/wiitm.2017.72324

\begin{abstract}
Introduction: Non-surgical minimally invasive treatments are greatly needed for patients with symptomatic benign prostatic hyperplasia (BPH), for whom medical treatment has failed and surgery is contraindicated. This study retrospectively evaluated the efficacy and safety of super-selective prostatic artery embolization (PAE) for BPH, relative to transurethral resection of the prostate (TURP).

Aim: To clinically evaluate the efficacy and safety of super-selective PAE for BPH, relative to TURP.

Material and methods: From February 2012 to March 2015, patients with BPH underwent selective PAE $(n=17)$ or TURP (control group; $n=40$ ). Prostate volume, maximum urinary flow rate (Qmax), International Prostate Symptoms Score (IPSS), and quality of life (QoL) score were evaluated at baseline and postoperative 3, 6, and 12 months. Complications were also recorded.

Results: All the procedures were technically successfully. At postoperative 1 year, patients given PAE had significantly greater prostate volume $(64.6 \pm 10.2 \mathrm{ml})$, IPSS $(23.9 \pm 4.9)$, and QoL $(4.1 \pm 0.7)$ compared with the control patients $(42.0 \pm 7.5 \mathrm{ml}, 13.1 \pm 3.5$, and $2.1 \pm 0.7$, respectively). The Qmax of the PAE group $(9.5 \pm 3.7 \mathrm{ml} / \mathrm{s})$ was significantly lower than that of the control $(21.8 \pm 4.2 \mathrm{ml} / \mathrm{s})$. The changes in parameters of the TURP patients relative to the preoperative baseline were significantly greater than those of the PAE group. No severe complications occurred.

Conclusions: Prostatic artery embolization was demonstrated as safe and effective and may be considered an alternative treatment for BPH patients, especially for those who are not candidates for or refuse surgery.
\end{abstract}

Key words: lower urinary tract symptoms, benign prostatic hyperplasia, transurethral resection of the prostate, super-selective prostatic artery embolization.

\section{Introduction}

Benign prostatic hyperplasia (BPH) is a very common disease in aging men and ordinarily leads to lower urinary tract symptoms (LUTS) [1, 2]. Although LUTS secondary to $\mathrm{BPH}$ are not usually life-threatening, they often compromise quality of life (QoL) [3]. Currently, the clinical management of symptomatic $\mathrm{BPH}$ is mainly medical, or surgical when medical treatment fails.
Transurethral resection of the prostate (TURP) has been the gold standard treatment for $\mathrm{BPH}$ for decades [4]. Nevertheless, TURP has been associated with significant postoperative complications, including incontinence or urinary retention, bleeding, and in some reports, sexual dysfunction such as retrograde ejaculation and impotence [5-7], especially in men older than 60 years [7]. Moreover, many symp- 
tomatic BPH patients are very elderly, and may have severe comorbidities that greatly increase the risks of performing TURP. Hence, non-surgical minimally invasive treatments are greatly needed as alternatives in the clinical management of BPH. Accordingly, several minimally invasive therapies have been proposed recently [8, 9].

Prostatic arterial embolization (PAE) was first introduced in the 1970s to control massive hemorrhage after prostatectomy or prostate biopsy $[10,11]$. Prostatic arterial embolization has proven to reduce prostatic volume and weight progressively when used to control spontaneous bleeding in BPH patients [12]. Increasing evidence has indicated that PAE is also a safe and effective technique for relief of LUTS in $\mathrm{BPH}$ patients $[13,14]$. In China, PAE by way of the superior vesical artery (super-selective PAE) remains a novel technology, and there are multiple challenges that prevent its wide application; clinical evidence supporting super-selective PAE as a primary treatment for LUTS due to BPH is quite limited $[15,16]$.

Herein, we retrospectively investigated the viability of PAE, with Embosphere microspheres as the embolic, as a primary treatment for LUTS in BPH patients who had failed medical treatment and who were unsuited for surgery. The therapeutic effect of super-selective PAE was evaluated relative to TURP during a follow-up of 1 year at our single center.

\section{Aim}

The aim was to conduct a clinical evaluation of the efficacy and safety of super-selective PAE for $\mathrm{BPH}$, relative to TURP.

\section{Material and methods}

The Ethics Committee of our hospital approved the protocol. The study was performed in accordance with ethical standards.

\section{Study participants}

Between February 2012 and March 2015, 17 BPH patients (mean age: $75.53 \pm 4.74$ years, range: 68 87 years), for whom medical treatment had failed and surgery was contraindicated, underwent super-selective PAE in our hospital. Each patient was assessed by a urologist and an anesthesiologist as being unsuited for surgery. The interventional radiologist was also involved in the patient selection.
The inclusion criteria for super-selective PAE were the following: a definitive diagnosis of $\mathrm{BPH}$ accompanied by severe LUTS; failure after 6-month administration of an $\alpha$-receptor blocker and $5 \alpha$-reductase inhibitors; concomitant severe cardiovascular and cerebrovascular diseases; chronic administration of anticoagulants that could not be terminated; lithotomy position was not possible due to limitation of the lower extremity; insertion of a resectoscope was not possible because of severe urethral stricture; and patient unwillingness to undergo the surgery.

Another $40 \mathrm{BPH}$ patients who underwent TURP (control group) were randomly selected by a random digits table from all hospitalized patients admitted to our hospital in the same period. These patients also had severe LUTS, but with no severe cardiovascular or cerebrovascular diseases.

The following preoperative (baseline) data of the PAE and control groups were compared: age, prostate volume, maximum urinary flow rate (Qmax), International Prostate Symptoms Score (IPSS) [17], and QoL score. Qmax was measured using uroflowmetry to analyze the quality of urination. The prostate of each patient was evaluated transrectally using ultrasound equipment (Mindray Biomedical Electronics, Shenzhen City, China). Prostate volume was calculated using the prostate ellipsoid formula (volume $=0.52 \times$ length $\times$ width $\times$ height $\mathrm{cm}$ ).

\section{Super-selective PAE procedures}

All the PAE procedures were performed by the same interventional radiologist (Dr. Kai Cheng), with 30 years of clinical experience in the field of interventional radiology. Specifically, after local anesthesia was achieved, the unilateral femoral artery was punctured using Seldinger's technique. A 4-5 Fr hydrophilic cobra-shaped catheter (Terumo, Tokyo, Japan) was inserted into the internal iliac arteries. Subsequently, pelvic angiography was performed by manually injecting 20-30 ml of contrast media (Ioversol) at $3 \mathrm{ml} / \mathrm{s}$ under fluoroscopic guidance.

During the procedure, the prostatic arterial supply was identified based on prostatic substance staining; the stained arteries revealed the outline of the prostate (Photo $1 \mathrm{~A}$ ). The prostatic artery at one side was selectively catheterized and Embosphere microspheres were used for embolization (90-180 $\mu \mathrm{m}$ in diameter; Merit Medical, Rockland, MA, USA). The microspheres were injected slowly under flu- 

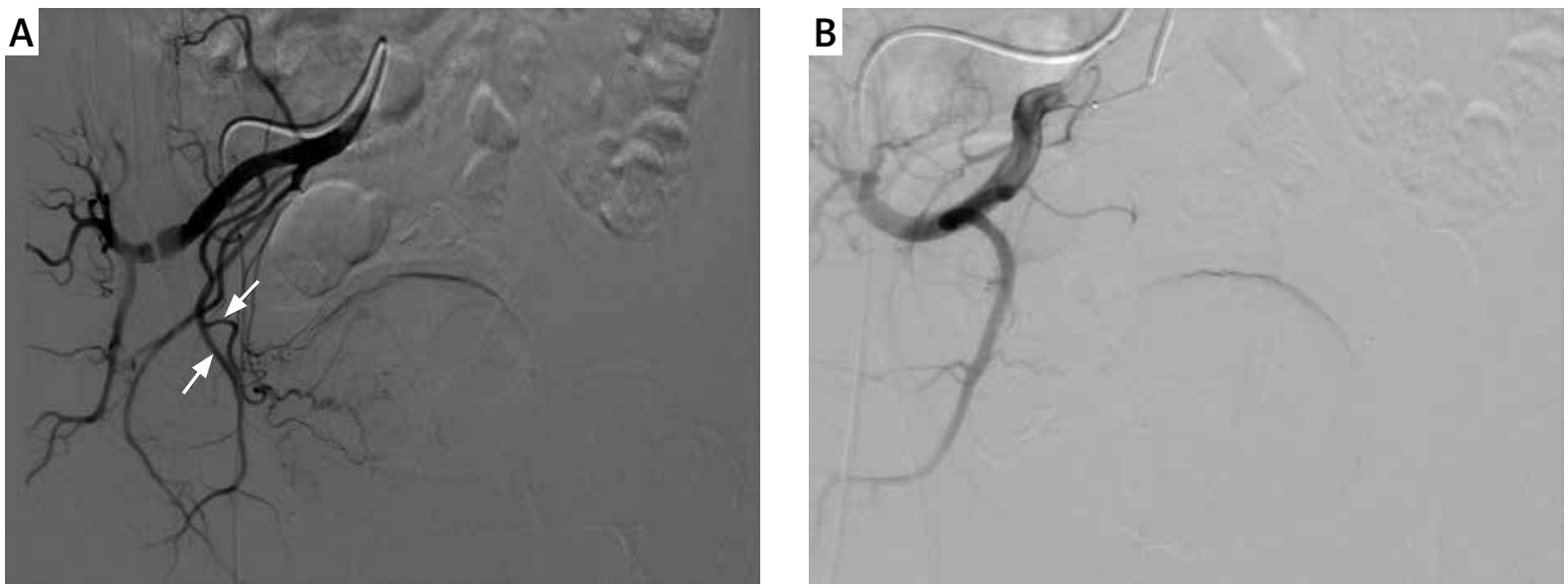

Photo 1. Right pelvic angiography of a 72-year-old male patient suffering from LUTS secondary to $\mathrm{BPH}$. A - The right prostate artery (down arrow) and internal pudendal artery (up arrow) before PAE. B - After PAE, the right prostate artery and internal pudendal artery are not seen

oroscopic guidance until the control angiogram revealed blood flow only in the proximal part of the prostatic artery, avoiding reflux of the embolic agent to undesired arteries. Follow-up angiography was conducted manually to check whether the distal arterial vasculature was dramatically depleted. Embolization was then performed on the contralateral side, using the same technique. Finally, all the catheters and sheaths were subsequently removed (Photo 1 B).

A radial compression device was placed over the puncture site for 20 min before bandaging. Infection prophylaxis was performed for every patient during the postoperative 3 days, and the urethral catheter was removed at postoperative 7 days.

\section{Transurethral resection of the prostate surgical procedures}

Transurethral resection of the prostate was conducted by the same urologist as the PAE (Dr. Zhi-Lei Qiu, with 10 years of clinical urologic experience). After epidural anesthesia was achieved, TURP was performed via transurethral electrocision under TV monitoring, using a standard Olympus isoionic resectoscope and bipolar generator. The resectoscope was inserted into the bladder under direct vision for determining the relationship between the bilateral ureteral opening, trigone of bladder, and the prostate intruded into the bladder. Also examined were the posterior urethra, seminal colliculus, and the shape and size of the prostatic hyperplasia. Layer-by-layer cutting of the prostate was performed, from the bladder neck to the seminal colliculus, to the prostatic capsule. Flusher (containing $37^{\circ} \mathrm{C}$ physiological saline) was used to draw off the excised prostatic gland. Finally, electrocoagulation hemostasis was performed, the resectoscope was removed, and the balloon catheter was left for 3 days postoperatively.

\section{Outcome measurements and follow-up methods}

Each patient in the PAE and TURP control groups was followed up at postoperative 3, 6, and 12 months by a designated physician. Functions were assessed by clinical observation of the prostate volume, Qmax, IPSS, and QoL scores. Other postoperative parameters observed during the follow-up included adverse events and postoperative complications.

\section{Statistical analysis}

Statistical analyses were performed using SPSS version 19.0 software (SPSS, Chicago, IL). Measurement data are expressed as mean \pm standard deviation. Intergroup comparisons were analyzed by the independent $t$-test. Intragroup comparisons between preoperative and postoperative data were analyzed by the paired $t$-test. $P<0.05$ was considered statistically significant.

\section{Results}

At the preoperative baseline, the PAE and TURP groups were statistically comparable with regard to 
Table I. Baseline demographics and clinical data of $\mathrm{BPH}$ patients undergoing super-selective PAE and TURP

\begin{tabular}{|lccl|}
\hline Parameter & PAE & TURP & $P$-value \\
\hline Subjects & 17 & 40 & \\
\hline Age [years] & $75.53 \pm 4.74$ & $73.35 \pm 4.75$ & 0.119 \\
\hline Prostatic volume $[\mathrm{ml}]$ & $64.6 \pm 10.2$ & $68.7 \pm 9.2$ & 0.146 \\
\hline Qmax [ml/s] & $9.5 \pm 3.7$ & $9.4 \pm 3.1$ & 0.962 \\
\hline IPSS & $23.9 \pm 4.9$ & $24.5 \pm 4.5$ & 0.672 \\
\hline QoL score & $4.1 \pm 0.7$ & $4.1 \pm 0.6$ & 0.723 \\
\hline
\end{tabular}

Table II. Origins of the prostatic artery among the 17 patients who underwent bilateral PAE

\begin{tabular}{|lcc|}
\hline Artery & $\begin{array}{c}\text { No. of } \\
\text { branches }\end{array}$ & $\begin{array}{c}\text { Prevalence } \\
\text { (\%) }\end{array}$ \\
\hline Inferior vesical artery & 19 & 48.7 \\
\hline Internal pudendal artery & 15 & 38.5 \\
\hline Internal iliac artery & 3 & 7.7 \\
\hline Obturator artery & 2 & 5.1 \\
\hline
\end{tabular}

Table III. Clinical data of functional parameters over time after PAE and TURP

\begin{tabular}{|c|c|c|c|c|}
\hline Parameter & & PAE & TURP & $P$ \\
\hline \multirow{4}{*}{$\begin{array}{l}\text { Prostate } \\
\text { volume } \\
{[\mathrm{ml}]}\end{array}$} & Baseline & $64.6 \pm 10.2$ & $68.7 \pm 9.2$ & 0.146 \\
\hline & 3 months & $52.3 \pm 5.9$ & $29.9 \pm 4.5$ & $<0.001$ \\
\hline & 6 months & $45.2 \pm 5.9$ & $30.8 \pm 5.2$ & $<0.001$ \\
\hline & 12 months & $42.0 \pm 7.5$ & $32.9 \pm 4.6$ & $<0.001$ \\
\hline \multirow{4}{*}{$\begin{array}{l}\text { Qmax } \\
{[\mathrm{ml} / \mathrm{s}]}\end{array}$} & Baseline & $9.5 \pm 3.7$ & $9.4 \pm 3.1$ & 0.962 \\
\hline & 3 months & $13.2 \pm 3.9$ & $20.4 \pm 4.4$ & $<0.001$ \\
\hline & 6 months & $16.4 \pm 4.5$ & $23.8 \pm 3.9$ & $<0.001$ \\
\hline & 12 months & $21.8 \pm 4.2$ & $24.3 \pm 3.7$ & 0.031 \\
\hline \multirow[t]{4}{*}{ IPSS } & Baseline & $23.9 \pm 4.9$ & $24.5 \pm 4.5$ & 0.672 \\
\hline & 3 months & $15.5 \pm 4.1$ & $12.3 \pm 3.5$ & 0.004 \\
\hline & 6 months & $12.1 \pm 3.4$ & $8.3 \pm 3.2$ & $<0.001$ \\
\hline & 12 months & $13.1 \pm 3.5$ & $10.2 \pm 4.5$ & 0.021 \\
\hline \multirow[t]{4}{*}{ QoL } & Baseline & $4.1 \pm 0.7$ & $4.1 \pm 0.6$ & 0.723 \\
\hline & 3 months & $2.8 \pm 1.1$ & $2.1 \pm 0.9$ & 0.010 \\
\hline & 6 months & $2.5 \pm 1.1$ & $1.9 \pm 0.9$ & 0.032 \\
\hline & 12 months & $2.1 \pm 0.7$ & $1.7 \pm 0.6$ & 0.034 \\
\hline
\end{tabular}

age, prostate volume, Qmax, IPSS, and QoL (Table I). No patient was lost to follow-up during the study period. The PAE was technically successful in 17 of 17 patients (100\%). The technical successful rate of TURP was also $100 \%$ (40/40) in the control group.

In the PAE group, each of the 17 patients underwent bilateral embolotherapy (Table II). Overall, angiography of 34 internal iliac arteries was completed in the PAE group. A sole artery supply was not found in any of these patients. Double, triple, and $\geq 4$ supply arteries were found in 4 (23.5\%), 5 (29.4\%), and $8(47.1 \%)$ patients, respectively. Embolotherapy was achieved on 39 branches of larger feeding arteries. Specifically, these included branches of 19 (48.7\%), 15 (38.5\%), 3 (7.7\%), and 2 (5.1\%) of inferior vesical, internal pudendal, internal iliac, and obturator arteries, respectively. Among these, the caliber of the inferior vesical arteries and internal pudendal arteries were the largest.

All patients were examined for prostate volume, Qmax, IPSS, and QoL score at each return follow-up visit (Table III). In the PAE group, the Qmax increased from the baseline $9.5 \pm 3.7 \mathrm{ml} / \mathrm{s}$, to $21.8 \pm 4.2 \mathrm{ml} / \mathrm{s}$ at postoperative 1 year, while there were significant reductions in the mean prostate volume (64.6 $\pm 10.2 \mathrm{ml}$ vs. $42.0 \pm 7.5 \mathrm{ml})$, IPSS $(23.9 \pm 4.9$ vs. 13.1 $\pm 3.5)$, and QoL score ( $4.1 \pm 0.7$ vs. $2.1 \pm 0.7$ ).

The postoperative pattern of changes in prostate volume, Qmax, IPSS, and QoL scores of the TURP patients relative to baseline was similar to that of the patients given PAE. However, compared with the PAE patients, in the TURP group the changes in prostate volume, Qmax, IPSS, and QoL scores at postoperative 3 months, 6 months, and 1 year were greater (Figure 1 A-D).

The operative time of PAE was 90 min (range: 70-120 min), and no intraoperative complications occurred. Among the 17 PAE patients, 14 achieved voluntary micturition after the removal of the urethral catheter at postoperative day 7, whereas $3(17.6 \%)$ patients were not able to urinate autonomously until the removal of the urethral catheter at postoperative day 14 . Within the first postoperative 7 days, 3 (17.6\%) and 5 (29.4\%) patients developed hypogastralgia (i.e., pain in the perineum, retropubic space, and/or urethra) and fever, respectively, which resolved quickly after symptomatic treatment. No severe complications occurred during the follow-up period, including vesical or intestinal ischemia or perforation. 
A

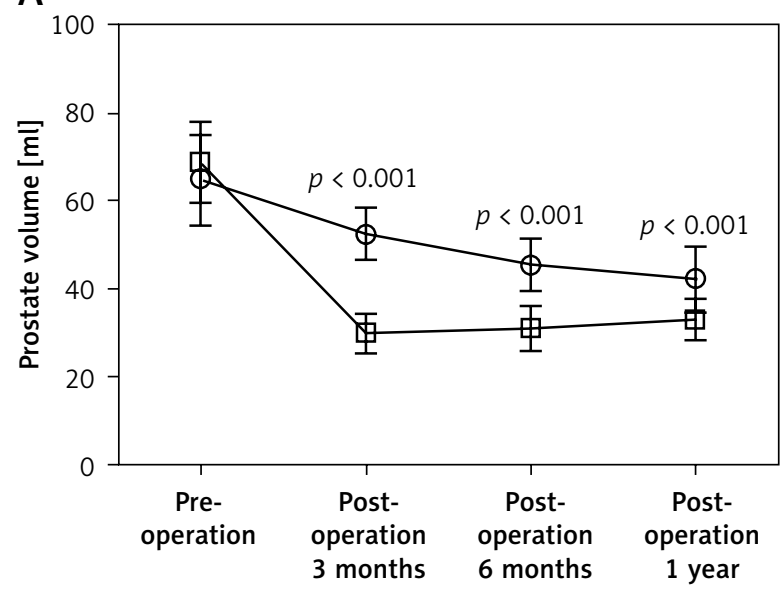

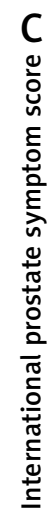

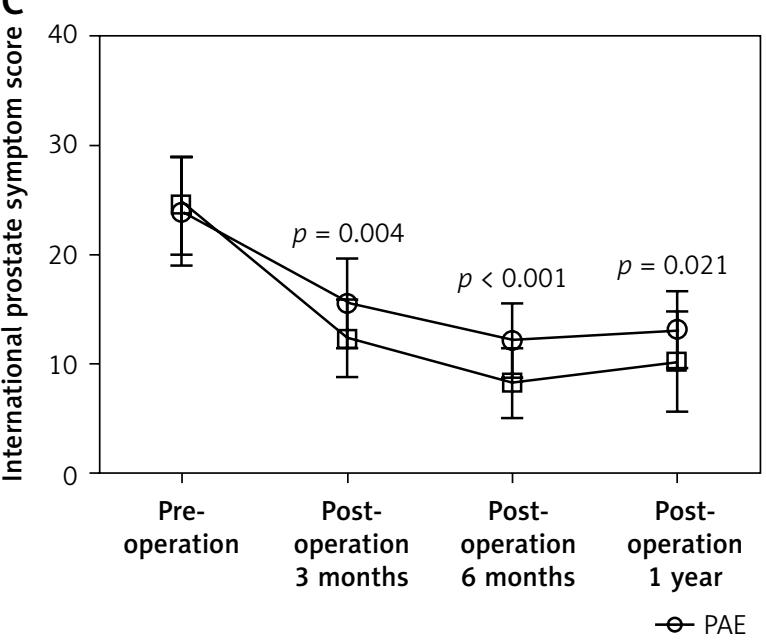

B

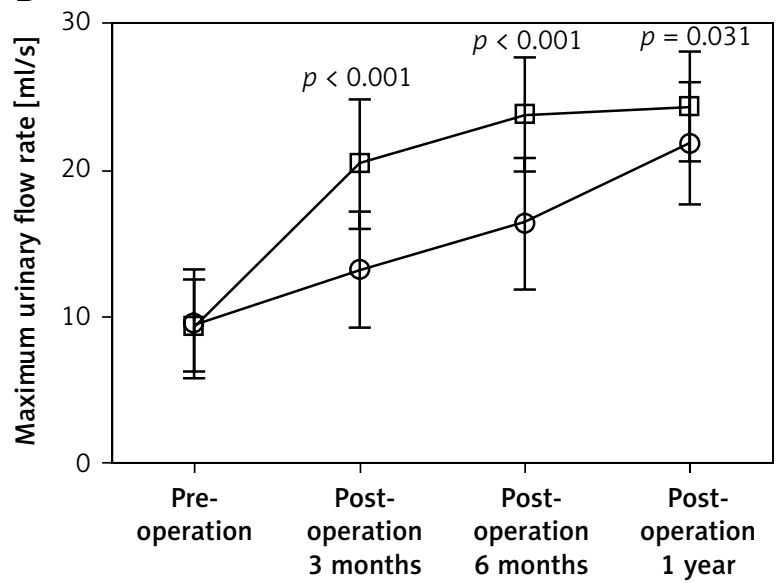

D

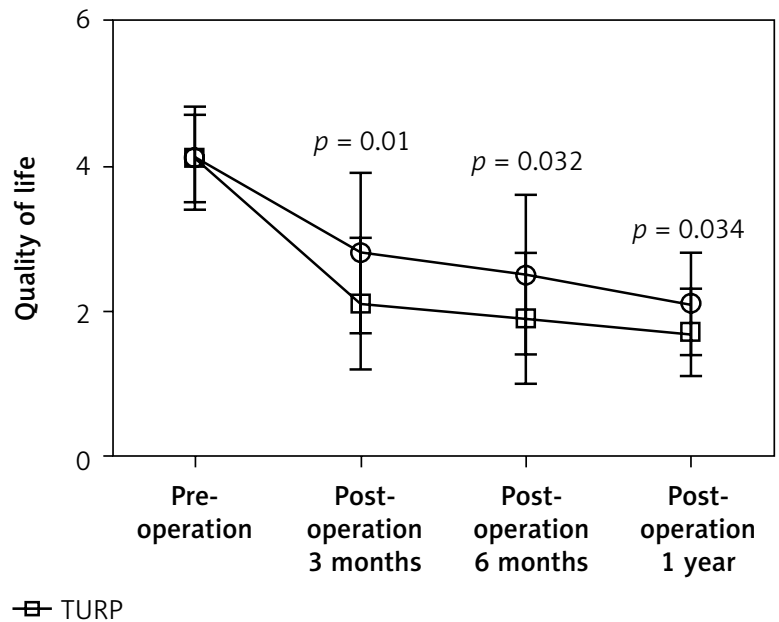

Figure 1. Comparisons of the functional parameters between PAE and TURP groups from baseline over time, including prostate volume (A), Qmax (B), IPSS (C), and QoL (D)

\section{Discussion}

Prostatic artery embolization for LUTS has been proposed as a minimally invasive alternative treatment for BPH [18-20]. As such, PAE is of great significance for $\mathrm{BPH}$ patients with concomitant severe diseases such as cardiovascular and cerebrovascular diseases and chronic obstructive pulmonary disease, and for whom surgery is contraindicated [21]. In this retrospective study, we compared the efficacy and safety of PAE with TURP for the treatment of BPH patients. We found that the LUTS of all the 17 patients were improved after the PAE procedure, as evidenced by increased Qmax, and decreased prostate volume, IPSS, and QoL scores, at the postoperative 3-, 6-, and 12-month follow-ups. Our results are consistent with previous studies by de Assis et al. [13] and Carnevale et al. [19], who reported prostate volume reduction $>30 \%$, mild symptoms, and improved QoL, 1 year after PAE. Collectively, these findings suggest that PAE is an effective treatment for patients with LUTS that are due to BPH.

The clinical results of bilateral PAE are putatively better than those of unilateral PAE [22]. Carnevale et al. [23] reported that 1 patient had unilateral PAE with continuous prostate reduction during the first postoperative year, but regrowth to the initial size was observed at 3 years. In our study, the patients who underwent super-selective bilateral PAE experienced significant prostate reduction during the first postoperative year. Evaluation of these patients is ongoing. 
In the patients given TURP, improvements in prostate volume, Qmax, IPSS, and QoL were superior to those of the PAE group, at all time points. This may be because, unlike TURP surgery, PAE is not immediately ablative. In theory, it may take more time after PAE for the prostate to undergo the histopathologic changes associated with destruction of the prostate vasculature $[24,25]$. In the present study, this was observed as lower differences in Qmax and IPSS, from baseline to postoperative 6 months- 1 year, of the PAE patients compared with the TURP group.

Complete understanding of the functional arterial anatomy is important for performing an effective and safe embolization, as it facilitates better clinical results, and avoids complications from untargeted embolization of the surrounding organs (bladder, rectum, and penis). In particular, the origin of the prostatic artery is highly variable among individuals [26]. Carnevale et al. [19] reported that the most common artery supplying the prostate was the inferior vesical artery. Consistent with this, the present study indicated that the most common artery supplying the prostate was the inferior vesical artery, followed by the internal pudendal arteries. However, Wang et al. [15] found that the most common artery supplying the prostate was the gluteal-pudendal trunk, followed by the superior vesical artery. They also reported that in $95.0 \%$ of cases the internal iliac artery had only one prostatic artery, and 5.1\% (11/218) had two independent prostatic arteries. Unlike these results, our data showed double arteries in 4 cases (23.5\%), three arteries in 5 cases (29.4\%), and more than three arteries in 8 cases (47.1\%). The differences may be attributable to the smaller sample size of the present study, or the ethnic origin of the study population. This is suggested by angiographic anatomical data reported by European physicians that may not be consistent with the actual situation in China [27].

In our study, all the PAE procedures were performed by the same interventional radiologist, who has 30 years of clinical experience in the field of interventional radiology at our hospital. The technical success rate was $100 \%$ among the 17 patients. Super-selective PAE still poses many technical challenges. In particular, accurately identifying the prostate artery is crucial, to avoid embolizing the surrounding normal blood vessels. In this study, accurate identification of the prostatic arterial supply was based on prostatic substance staining; the arteries surround- ing the prostate were stained to display its outline. If there is no feeding artery directly from the internal iliac artery, the superior prostate supply artery should include the inferior vesical, internal pudendal, inferior rectal, or obturator arteries [28]. In the present study, although there were numerous feeding arteries to the prostate, in nearly all BPH patients there was a predominant feeding artery that should be preferred for arterial embolization, as it is generally larger in diameter and the catheter is easier to insert. We believe that the best therapeutic effect is achieved by embolization of the bilateral prostate arteries and other blood-supply branches. Thus the efficacy should persist, and collateral formation is avoided, reducing prostate regeneration and symptomatic relapse.

There is limited information regarding the embolization materials to be used in PAE procedures. The Embosphere microspheres used in the present study are among the latest generation of embolic materials [29]. These are made of acrylic polymer, with an outer layer of hydrophilic gelatin. The advantages include flow with the bloodstream to effect complete vascular luminal blockage, accurate targeting, and peripheral embolization. Another important component of PAE that remains to be determined is the optimal embolic agent size, which should be investigated.

The present study is limited by its retrospective and non-randomized nature. In addition, the number of patients treated with PAE was not large or sufficient, and the follow-up time was not long enough to evaluate long-term clinical outcomes. Nevertheless, this study shows that super-selective PAE with microspheres can be a safe and efficient treatment for very elderly patients with $\mathrm{BPH}$, and especially for patients who are unsuited for or intolerant of TURP.

\section{Conclusions}

The present clinical data showed that both super-selective PAE and TURP resulted in significant clinical improvements in the treatment of LUTS due to BPH. TURP showed superior clinical improvements at all follow-up time points compared with PAE. Prostatic artery embolization seems to be safe and effective for patients with severe LUTS due to $\mathrm{BPH}$, and may be considered an alternative treatment for symptomatic BPH patients. This is espe- 
cially true of patients for whom medical treated has failed, who are not candidates for surgery, or who refuse surgery.

\section{Acknowledgments}

We are grateful to the reviewers and editors for their valuable and helpful comments on this paper.

The study was supported by the Medical Guidance Program of Qingdao City (2015-WJZD002) and Qingdao Outstanding Health Professional Development Fund.

\section{Conflict of interest}

The authors declare no conflict of interest.

\section{References}

1. Thorpe A, Neal D. Benign prostatic hyperplasia. Lancet 2003 361: 1359-67.

2. Oelke M, Bachmann A, Descazeaud A, et al. EAU guidelines on the treatment and follow-up of non-neurogenic male lower urinary tract symptoms including benign prostatic obstruction. Eur Urol 2013; 64: 118-40.

3. McVary KT, Roehrborn CG, Avins AL, et al. Update on AUA guideline on the management of benign prostatic hyperplasia. J Uro 2011; 185: 1793-803.

4. Pinheiro LC, Martins Pisco J. Treatment of benign prostatic hyperplasia. Tech Vasc Interv Radiol 2012; 15: 256-60.

5. Reich O, Gratzke C, Bachmann A, et al. Morbidity, mortality and early outcome of transurethral resection of the prostate: a prospective multicenter evaluation of 10,654 patients. J Urol 2008; 180: 246-9.

6. Favilla V, Cimino S, Salamone C, et al. Risk factors of sexual dysfunction after transurethral resection of the prostate (TURP) a 12 months follow-up. J Endocrinol Invest 2013; 36: 1094-8.

7. Rassweiler J, Teber D, Kuntz R, et al. Complications of transurethral resection of the prostate (TURP): incidence, management, and prevention. Eur Urol 2006; 50: 969-79.

8. Denmeade SR, Egerdie B, Steinhoff G, et al. Phase 1 and 2 studies demonstrate the safety and efficacy of intraprostatic injection of PRX302 for the targeted treatment of lower urinary tract symptoms secondary to benign prostatic hyperplasia. Eur Urol 2011; 59: 747-54.

9. Lourenco T, Pickard R, Vale L, et al. Minimally invasive treatments for benign prostatic enlargement: systematic review of randomised controlled trials. BMJ 2008; 337: a1662.

10. Mitchell ME, Waltman AC, Athanasoulis CA, et al. Control of massive prostatic bleeding with angiographic techniques. J Urol 1976; 115: 692-5.

11. Michel F, Dubruille T, Cercueil JP, et al. Arterial embolization for massive hematuria following transurethral prostatectomy. J Urol 2002; 168: 2550-1.

12. DeMeritt JS, Elmasri FF, Esposito MP, et al. Relief of benign pros tatic hyperplasia-related bladder outlet obstruction after trans arterial polyvinyl alcohol prostate embolization. J Vasc Interv Radiol 2000; 11: 767-70.

13. de Assis AM, Moreira AM, de Paula Rodrigues VC, et al. Prostatic artery embolization for treatment of benign prostatic hyperplasia in patients with prostates $>90 \mathrm{~g}$ : a prospective single-center study. J Vasc Interv Radiol 2015; 26: 87-93.

14. Pisco JM, Pinheiro LC, Bilhim T, et al. Prostatic arterial embolization to treat benign prostatic hyperplasia. J Vasc Interv Radiol 2011; 22: 11-9.

15. Wang MQ, Guo LP, Zhang GD, et al. Prostatic arterial embolization for the treatment of lower urinary tract symptoms due to large (>80 mL) benign prostatic hyperplasia: results of midterm follow-up from Chinese population. BMC Urol 2015; 15: 33.

16. Gao YA, Huang Y, Zhang R, et al. Benign prostatic hyperplasia: prostatic arterial embolization versus transurethral resection of the prostate: a prospective, randomized, and controlled clinical trial. Radiology 2014; 270: 920-8.

17. Barry MJ, Fowler FJ Jr, O'Leary MP, et al. The American Urological Association symptom index for benign prostatic hyperplasia. The Measurement Committee of the American Urological Association. J Urol 1992; 148: 1549-57.

18. Carnevale FC, Antunes AA, Prostatic artery embolization for enlarged prostates due to benign prostatic hyperplasia. How I do it. Cardiovasc Intervent Radiol 2013; 36: 1452-63.

19. Carnevale FC, da Motta-Leal-Filho JM, Antunes AA, et al. Quality of life and clinical symptom improvement support prostatic artery embolization for patients with acute urinary retention caused by benign prostatic hyperplasia. J Vasc Interv Radiol 2013; 24: 535-42.

20. Rio Tinto H, Martins Pisco J, Bilhim T, et al. Prostatic artery embolization in the treatment of benign prostatic hyperplasia: short and medium follow-up. Tech Vasc Interv Radiol 2012; 15: 290-3.

21. Baazeem A, Elhilali MM. Surgical management of benign prostatic hyperplasia: current evidence. Nat Clin Pract Urol 2008; 5: 540-9.

22. Bilhim T, Pisco J, Rio Tinto $\mathrm{H}$, et al. Unilateral versus bilateral prostatic arterial embolization for lower urinary tract symptoms in patients with prostate enlargement. Cardiovasc Intervent Radiol 2013; 36: 403-11.

23. Carnevale FC, da Motta-Leal-Filho JM, Antunes AA, et al. Midterm follow-up after prostate embolization in two patients with benign prostatic hyperplasia. Cardiovasc Intervent Radiol 2011; 34: 1330-3.

24. Sun F, Sánchez FM, Crisóstomo V, et al. Transarterial prostatic embolization: initial experience in a canine model. AJR Am J Roentgenol 2011; 197: 495-501.

25. Sun F, Sánchez FM, Crisóstomo V, et al. Benign prostatic hyperplasia: transcatheter arterial embolization as potential treatment: preliminary study in pigs. Radiology 2008; 246: 783-9.

26. Bilhim T, Tinto HR, Fernandes $L$, et al. Radiological anatomy of prostatic arteries. Tech Vasc Interv Radiol 2012; 15: 276-85.

27. Schreuder SM, Scholtens AE, Reekers JA, et al. The role of prostatic arterial embolization in patients with benign prostatic hyperplasia: a systematic review. Cardiovasc Intervent Radiol 2014: 37: 1198-219. 
28. Bilhim T, Pisco JM, Rio Tinto H, et al. Prostatic arterial supply: anatomic and imaging findings relevant for selective arterial embolization. J Vasc Interv Radiol 2012; 23: 1403-15.

29. Verret V, Ghegediban SH, Wassef M, et al. The arterial distribution of Embozene and Embosphere microspheres in sheep kidney and uterus embolization models. J Vasc Interv Radiol 2011; 22: 220-8.

Received: 30.07.2017, accepted: 17.10.2017. 